Document downloaded from:

http://hdl.handle.net/10251/63984

This paper must be cited as:

Baselga Moreno, S. (2015). Application of robust estimation methods to simple models of nucleon separation energies. Modern Physics Letters A. 30(24):1550121-1-1550121-12. doi:10.1142/S0217732315501217.

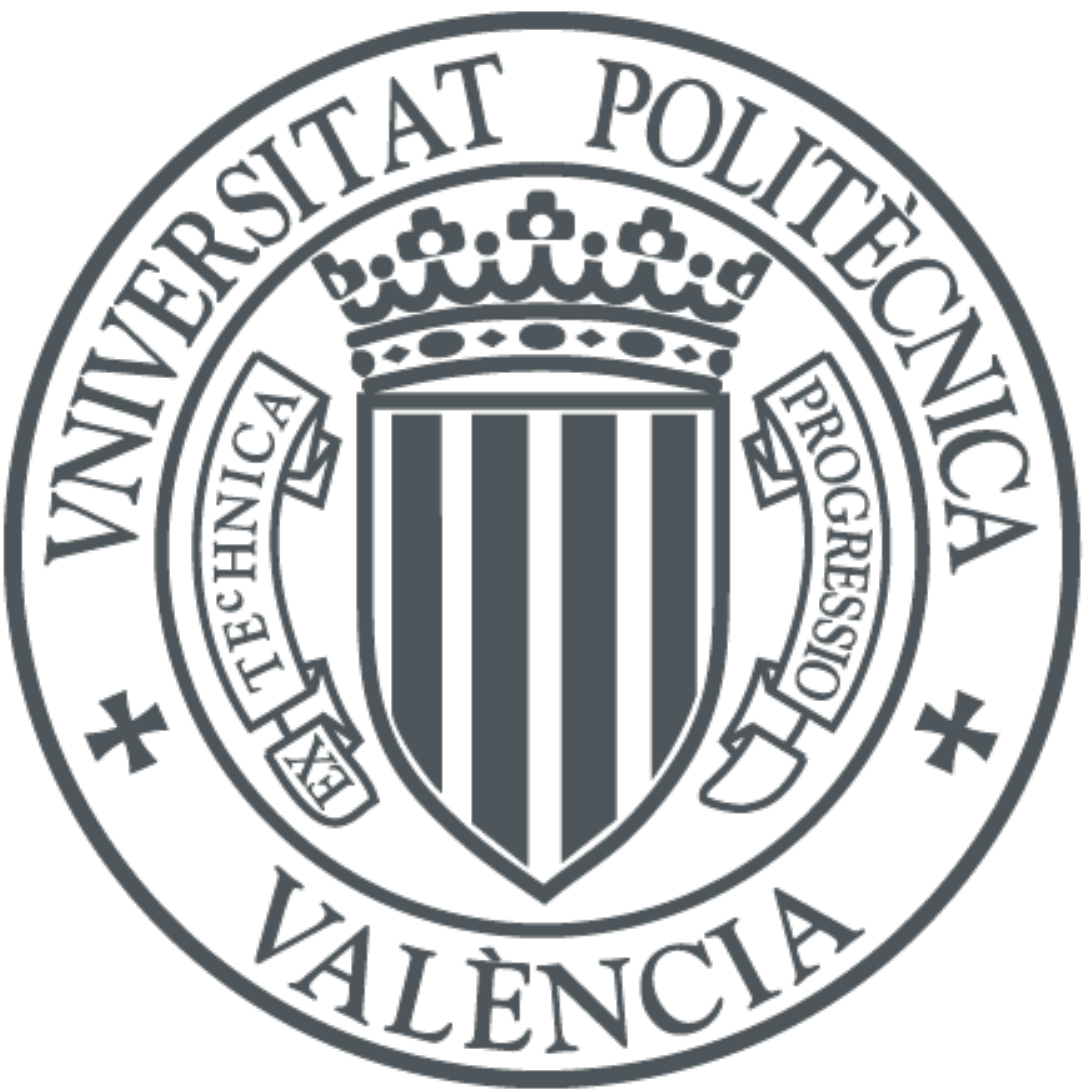

The final publication is available at

http://dx.doi.org/10.1142/S0217732315501217

Copyright World Scientific Publishing

Additional Information 


\title{
Application of robust estimation methods to simple models of nucleon separation energies
}

\author{
S Baselga \\ Universidad Politécnica de Valencia, Camino de Vera s/n, 46022 Valencia, Spain. \\ E-mail: serbamo@cgf.upv.es
}

\begin{abstract}
Some works have recently shown the usefulness of simple models of nucleon separation energies in terms of neutron and proton numbers. However, the customary use of least squares in the process of parameter estimation turns out to be extremely sensible to the accuracy of the model and the extent and quality of data (e.g. highly vulnerable to the sample size or the possible existence of undesired errors in the experimental values). We will show how robust estimation by global optimization instead of least squares estimation improves on both the stability of the estimated parameters and the extrapolation to unknown energies. Comparison against recently determined experimental data will show a level of agreement comparable to the predictions made by the best and much more complex models.
\end{abstract}

Keywords: separation energy; nuclear structure models; robust estimation; global optimization

PACS: 21.10.Dr; 14.20.Dh; 21.60.-n

\section{Introduction}

Nucleon separation energies are fundamental properties of the atomic nucleus. They play an essential role in nuclear structure theory and nuclear astrophysics, e.g. in the rapid proton and rapid neutron capture processes. Therefore they have been the subject of extensive research in recent years (for a comprehensive review see e.g. [1]).

A number of models and relations have been proposed for nucleon separation energies (or for nuclear masses or binding energies), among others, the Weizsäcker and improved Weizsäcker models [2-3], the Galvey-Kelson mass relations [4-6], the Duflo-Zuker model [7] and mass relations in terms of proton-neutron interactions [8,9]. Most of the formulas currently used involve extensive theoretical calculations and depend on so many parameters that a possible dominant dependence on simple physical quantities like neutron and proton numbers, $N$ and $Z$, is obscured. Recently, some studies have shown the usefulness of very simple models of nucleon separation energies in terms of $N / Z$ (or $Z / N$ ) [10,11]. Their agreement with experimental data is fairly good and therefore they can be proposed to be used for extrapolation to unmeasured separation energies. In the present paper we show, however, that the model parameters obtained after a least squares adjustment are extremely sensible to the particular data used in the adjustment and that extrapolation to unknown separation energies, although to a lesser degree, is also considerably dependent on them. One may argue that the proposed simple models are too simplistic to encapsulate all the nuclear processes involved and therefore predictions based on them shall not be much accurate. However, we will show how the use of a robust estimation method instead of the least squares estimator significantly improves the stability of the estimated parameters and extrapolations to unknown separation energies. As we were initially motivated by Ref. [10] we used the same dataset, i.e. the nucleon separation energies compilation of Audi and Wapstra [12], in order to reproduce, first, and improve, second, their results. Since several of the then unknown separation energies have already been measured at present we were also able to test the respective predictions of Audi and Wapstra, and the simple model with parameters obtained both by means of a least squares adjustment and by robust estimation. Results demonstrate the best performance of the latter. 


\section{Methods}

\subsection{Simple model for nucleon separation energies}

If we denote by $S_{n}$ the one-neutron separation energy we can write, according to [10],

$$
S_{n}=\left(a_{1}+a_{2} A^{1 / 3}\right) \frac{Z}{N}-a_{3}+\delta_{\text {pair }}-\delta_{\text {shell }}
$$

with constant parameters $a_{i}$ for all nuclei, where $A$ is the mass number, $\delta_{\text {pair }}$ is a pairing term of the form usually found in Weizsäcker type formulas

$$
\delta_{\text {pair }}=\left\{\begin{array}{lc}
+a_{\text {pair }} / A^{1 / 2}, & N \text { even } \\
-a_{\text {pair }} / A^{1 / 2}, & N \text { odd }
\end{array}\right.
$$

and

$$
\delta_{\text {shell }}=a_{\text {shell }} \cdot n(N)
$$

$$
n(N)=\left\{\begin{array}{lr}
0, & 1 \leq N \leq 28 \\
1, & 29 \leq N \leq 50 \\
2, & 51 \leq N \leq 82 \\
3, & 83 \leq N \leq 126 \\
4, & 127 \leq N
\end{array}\right.
$$

which accounts for the main part of the shell correction, the discontinuity at the magic neutron numbers.

For the one-proton separation energy, $S_{p}, N$ and $Z$ have to be interchanged and an additional Coulomb term is needed [10]. Therefore we can write

$$
S_{p}=\left(a_{1}+a_{2} A^{1 / 3}\right) \frac{N}{Z}-a_{3}+\delta_{\text {Coulomb }}+\delta_{\text {pair }}-\delta_{\text {shell }}
$$

where $a_{i}$ are constant parameters (different from those in Eq. 1) for all nuclei and

$$
\begin{aligned}
& \delta_{\text {Coulomb }}=-a_{\text {Coulomb }} \cdot Z / A^{1 / 3} \\
& \delta_{\text {pair }}= \begin{cases}+a_{\text {pair }} / A^{1 / 2}, & Z \text { even } \\
-a_{\text {pair }} / A^{1 / 2}, & Z \text { odd }\end{cases} \\
& \delta_{\text {shell }}=a_{\text {shell }} \cdot n(Z)
\end{aligned}
$$

$$
n(Z)=\left\{\begin{array}{rr}
0, & 1 \leq Z \leq 28 \\
1, & 29 \leq Z \leq 50 \\
2, & 51 \leq Z \leq 82 \\
3, & 83 \leq Z \leq 126 \\
4, & 127 \leq Z
\end{array}\right.
$$




\subsection{Robust estimation by global optimization}

It is well known that the least squares estimator is the best estimator (with some desirable properties like unbiasedness and linearity) for the cases where only Gaussian errors occur, i.e. where there are neither gross errors in the observations nor systematic errors in the model (or observations).

Let it be the following over-determined system of equations

$$
A x=l+v
$$

where $\boldsymbol{A}$ is the coefficient matrix for the vector of unknowns $\boldsymbol{x}, \boldsymbol{l}$ is the observation vector and $\boldsymbol{v}$ is the vector of residuals. Then the least squares solution is the one that minimizes the sum of squared residuals

$$
\boldsymbol{x}=\arg \min \sum v_{i}^{2}
$$

which is readily obtained by

$$
\boldsymbol{x}=\left(\boldsymbol{A}^{T} \boldsymbol{A}\right)^{-1} \boldsymbol{A}^{T} \boldsymbol{l}
$$

However, it turns out that this solution may be extremely sensible to the accuracy of the functional model (in our case, Eq. (1) for $S_{n}$ or Eq. (5) for $S_{p}$ ) and the quality of data (i.e. the possible existence of undesired errors in the sample of $S_{n}$ or $S_{p}$ values).

Alternatively to least squares estimation, robust estimation was devised for the cases were deviations from the theoretical functional model or from the observation statistical model may be expected [13]. Some of the ideas concerning robust estimation can be traced back to the 18th century and found in the works of Boscovich and Maire, Laplace and other contemporaries [14-15] which already proposed the $\mathrm{L}_{1}$ norm that we use in this paper. The foundations of the modern theory of robust estimation were established by Box [16] and developed by some other later contributors (Andrews et al. [17], Huber [18], etc).

Robust estimation is based on minimizing a function - called the estimator - in order to attain a solution that is maximum resistant to the non-fulfilment of the functional model or the appearance of gross or systematic errors in the observations. If the model is correct and the observations are affected only by random errors then robust estimation yields a solution close to that of classic least squares estimation. By contrast, if systematic or gross errors affect the observations or the model is not completely correct then robust estimation provides the most accurate solution whereas least squares, which is much less robust, yields a highly contaminated and unacceptable solution.

Among all the robust estimators, we will focus on one of the most successful, the $\mathrm{L}_{1}$ norm, the use of which is widespread in the literature, e.g. [19-24]. For the system of equations defined in Eq. (10), the least $L_{1}$ norm (or least absolute deviation) solution is the one that minimizes the sum of absolute values of residuals

$$
\boldsymbol{x}=\arg \min \sum\left|v_{i}\right|
$$

The typical procedure to compute such an estimator is an iteratively reweighed least-squares (IRLS) scheme, by which the estimator minimization - Eq. (13) - is performed by successive least squares adjustments computing for each of them equivalent weights based on the previous adjustment residuals. The equivalent weight function for the $\mathrm{L}_{1}$ norm estimator to be used within least-squares is 
$w_{i}=\frac{1}{\left|v_{i}\right|}$

So that the solution to the weighted least squares system yields the $\mathrm{L}_{1}$ norm minimization

$$
\boldsymbol{x}=\arg \min \sum v_{i}^{2} w_{i}=\arg \min \sum v_{i}^{2} \frac{1}{\left|v_{i}\right|}=\arg \min \sum\left|v_{i}\right|
$$

However, as we showed previously [22], the problem with robust estimation as IRLS is a computational one, for if the initial least-squares solution lies far away from the correct solution then the iterative process started with it attains only a local optimum. Therefore we will deal with robust estimation as a global optimization problem in order to ensure attainment of the global optimum.

Let us recast the question in the form of a global optimization problem

$$
\left\{\begin{array}{l}
\min f \\
\text { being } f(\boldsymbol{v})=\sum\left|v_{i}\right| \quad / \boldsymbol{A x}=\boldsymbol{l}+\boldsymbol{v} \\
\text { for } x_{i} \in D
\end{array}\right.
$$

where $f$ is the objective function and $x_{i}$ are the variables to optimize within a search domain $D$.

Among the vast variety of global optimization methods, which can be classified into heuristic and deterministic methods [25], we will use the Simulated Annealing (SA) method. It is an iterative heuristic method based in the analogy with the self-construction process of crystalline networks, which was originally proposed by Metropolis et al [26], and later developed by Kirkpatrick et al [27], among many others. It was the first method to succeed in solving the travelling salesman problem (i.e. the shortest itinerary for a salesman to visit a given set of cities). We only sketch here a simple procedure for application. For further details please refer to more specific literature, e.g. [28-29].

The method tries to emulate the formation process of a crystalline network; therefore, three key features have to be implemented:

1. The thermal agitation of particles, directly dependent on temperature (iteration, in our analogy). It is customary to select a zero-centred Gaussian distribution, i.e.

$$
\Delta x_{i} \sim N\left(0, \sigma^{(i)}\right)
$$

with corresponding probability density function

$$
\operatorname{pdf}\left(\Delta x_{i}\right)=\frac{1}{\sqrt{2 \pi} \sigma^{(i)}} e^{-\frac{\Delta x_{i}^{2}}{2 \sigma^{(i)^{2}}}}
$$

2. The cooling scheme, responsible for the amplitude reduction of thermal agitation. With the temperature decrease (the course of iterations in our analogy) displacements - Eq. (17) undergo a gradual amplitude decrease. A simple though successful expression is

$$
\sigma^{(i)}=\beta^{i} \sigma^{(0)}
$$

where $\sigma^{(0)}$ is the initial amplitude (a value that permits to comfortably explore the search domain width in few steps) and $\beta$ is the cooling factor, whose value has to be selected 
according to the problem complexity. See the next section for the particular values used for $\sigma^{(0)}$ and $\beta$ in our problem at hand.

3. The acceptance criteria, used to decide whether to accept or reject the solution proposed in the iteration on the basis of the particular value of the objective function. The acceptance criteria for the particular iteration $j$ can be written as

$$
\boldsymbol{x}^{(j)}=\left\{\begin{array}{l}
\boldsymbol{x}^{(j-1)}+\Delta \boldsymbol{x}^{(j)} \quad \text { if } f\left(\boldsymbol{x}^{(j-1)}+\Delta \boldsymbol{x}^{(j)}\right)<f\left(\boldsymbol{x}^{(j-1)}\right) \\
\boldsymbol{x}^{(j-1)}+\Delta \boldsymbol{x}^{(j)} \quad \text { otherwise, with probability } p \\
\boldsymbol{x}^{(j-1)} \quad \text { otherwise }
\end{array}\right.
$$

where $p$ is a small number (e.g. 0.001) that defines the probability of accepting a solution that is worse than the previous one and whose inclusion contributes to preventing the algorithm from getting stuck into local minima.

Therefore, the scheme to solve our robust estimator - minimum $\mathrm{L}_{1}$ norm, Eq. (16) - by SA can be summarized as:

Step 1. Take an arbitrary initial solution for the vector to estimate $\boldsymbol{x}^{(0)}$ (its value is not critical, it can be e.g. the result of a prior least squares adjustment) and compute by Eq. (16) the corresponding value for the objective function $f\left(x^{(0)}\right)$.

Step 2. Generate a vector of displacements $\Delta \boldsymbol{x}$ by using the thermal agitation function (17) in every vector component, using the standard deviation for the corresponding iteration in accordance with the cooling scheme (19).

Step 3. Accept or reject this solution as the basis for the next iteration as decided by the acceptance criteria (20).

Return to step 2 to perform the next iteration until a finishing criterion is fulfilled. A simple finishing criterion may be to reach a standard deviation for the iteration $\sigma^{(\mathrm{i})}$ that is below the desired degree of accuracy in the solution.

The convergence to the global optimum in a finite number of iterations is guaranteed in probabilistic terms [28] and depends mainly on the cooling scheme: if cooling is too fast, i.e. if $\beta$ in (19) makes the amplitude drop too quickly, the final state is an amorphous one (a local minimum) and not the crystalline state (the global minimum). Conversely, if cooling is too slow, i.e. if $\beta$ in (19) makes the amplitude diminish too slowly, then the computation time becomes uncomfortably high. $\beta, \sigma^{(0)}$ and $\sigma^{(\text {final) }}$ are parameters that have to be tuned for the particular problem (see the next section for the particular values used in our problem).

\section{Results and discussion}

Since we were inspired in this paper by the simple model in [10] we used here the same dataset of nucleon separation energies, i.e. the compilation by Audi and Wapstra [12]. We are well aware that newer datasets have been published since then, particularly the AME2003 [30] and AME2012 [31] databases; however, we will use the same data as in [10] since we are interested now in showing the improvement, both in accuracy and stability, attained by using robust estimation as a global optimization problem instead of least squares.

Once these advantages have become evident we will end up by testing the quality of predictions for unmeasured energies. Since several of the then unknown separation energies have already been measured at present (and available in [31]) we will be able to test the respective predictions of Audi 
and Wapstra, and the simple model with parameters obtained both by means of a least squares adjustment and by robust estimation. Results will demonstrate the best performance of our method.

Analogously to [10] we used the so-called recommended values in [12] (file rct2_rmd.mas95) and omitted all data points which have not been measured but derived from systematic trends as well as all nuclei with $A \leq 40$.

We performed the least squares (LS) adjustment of one-neutron separation energies - following Eq. (1) - and one-proton separation energies - following Eq. (5) - and obtained the same results as [10]. We also obtained both sets of parameters by means of robust estimation (RE) computed by the SA method as explained in the previous section, with the following values for tuneable parameters:

- Starting parameters $x^{(0)}$ : least squares solution.

- Search domain $D$ : least squares solution $\pm 20000 \mathrm{keV}$ for each unknown (this search domain can be made arbitrarily wider at the only cost of additional computational burden).

- Initial amplitude $\sigma^{(0)}=200 \mathrm{keV}$, one or two orders lower than the search domain width so that it can explore it comfortably in some hundred or thousand iterations.

- Final amplitude $\sigma^{(\text {final })}=0.2 \mathrm{keV}$, since variations of this order are completely negligible for our purposes.

- Small probability $p=0.001$, in the acceptation of worse solutions, only to help escape from local minima.

- Cooling factor $\beta=0.9999$, since along with the rest of the parameters it provides a reliable and relatively fast estimation procedure (with computing time of the order of a couple of seconds in a conventional personal computer).

The process resulted in 69075 iterations and the parameters obtained for both the one-neutron and one-proton separation energies are displayed along with the ones estimated by least squares in Table 1 and Table 2. It is worth noting that repeated executions of the algorithm provide slightly different sets of parameters whose differences are negligible for our purposes.

Table 1. Parameters for $S_{n}$ after LS and RE adjustments. Root mean square (rms) deviations and residual percentile are also provided.

\begin{tabular}{ccccccccccccc}
\hline & $\begin{array}{c}a_{1} \\
(\mathrm{keV})\end{array}$ & $\begin{array}{c}a_{2} \\
(\mathrm{keV})\end{array}$ & $\begin{array}{c}a_{3} \\
(\mathrm{keV})\end{array}$ & $\begin{array}{c}a_{\text {pair }} \\
(\mathrm{keV})\end{array}$ & $\begin{array}{c}a_{\text {shell }} \\
(\mathrm{keV})\end{array}$ & $\begin{array}{c}\mathrm{rms} \\
(\mathrm{keV})\end{array}$ & $\begin{array}{c}v_{0.99} \\
(\mathrm{keV})\end{array}$ & $\begin{array}{c}v_{0.95} \\
(\mathrm{keV})\end{array}$ & $\begin{array}{c}v_{0.90} \\
(\mathrm{keV})\end{array}$ & $\begin{array}{c}v_{0.80} \\
(\mathrm{keV})\end{array}$ & $\begin{array}{c}v_{0.75} \\
(\mathrm{keV})\end{array}$ & $\begin{array}{c}v_{0.50} \\
(\mathrm{keV})\end{array}$ \\
\hline$S_{n}(\mathrm{LS})$ & 6294 & 3433 & 5850 & 10587 & 1512 & 423 & 1364 & 813 & 652 & 501 & 445 & 268 \\
$S_{n}(\mathrm{RE})$ & 5227 & 3532 & 5364 & 10874 & 1552 & 429 & 1456 & 811 & 646 & 485 & 440 & 260 \\
\hline
\end{tabular}

Table 2. Parameters for $S_{p}$ after LS and RE adjustments. Root mean square (rms) deviations and residual percentile are also provided.

\begin{tabular}{cccccccccccccc}
\hline & $\begin{array}{c}a_{1} \\
(\mathrm{keV})\end{array}$ & $\begin{array}{c}a_{2} \\
(\mathrm{keV})\end{array}$ & $\begin{array}{c}a_{3} \\
(\mathrm{keV})\end{array}$ & $\begin{array}{c}a_{\text {Coulomb }} \\
(\mathrm{keV})\end{array}$ & $\begin{array}{c}a_{\text {pair }} \\
(\mathrm{keV})\end{array}$ & $\begin{array}{c}a_{\text {shell }} \\
(\mathrm{keV})\end{array}$ & $\begin{array}{c}\mathrm{rms} \\
(\mathrm{keV})\end{array}$ & $\begin{array}{c}v_{0.99} \\
(\mathrm{keV})\end{array}$ & $\begin{array}{c}v_{0.95} \\
(\mathrm{keV})\end{array}$ & $\begin{array}{c}v_{0.90} \\
(\mathrm{keV})\end{array}$ & $\begin{array}{c}v_{0.80} \\
(\mathrm{keV})\end{array}$ & $\begin{array}{c}v_{0.75} \\
(\mathrm{keV})\end{array}$ & $\begin{array}{c}v_{0.50} \\
(\mathrm{keV})\end{array}$ \\
\hline$S_{p}(\mathrm{LS}) 13831$ & 636 & 3557 & 982 & 12141 & 1319 & 461 & 1481 & 793 & 665 & 531 & 473 & 292 \\
$S_{p}(\mathrm{RE})$ & 12179 & 863 & 1917 & 1068 & 12387 & 1312 & 467 & 1659 & 795 & 650 & 512 & 458 & 283 \\
\hline
\end{tabular}

The first evident observation is that the model parameters for $S_{n}$ (or $S_{p}$ ), $a_{1}, a_{2}$, etc are quite different depending on whether they are estimated by LS or RE. At any rate, apart from the possible physical significance, those differences do not have a very large impact on the respective predictions that can be made from them, as we will show later. 
Secondly, regarding the statistic parameters, the root mean square (rms) deviation is very slightly larger for the RE adjustment, 429 as compared to $423 \mathrm{keV}$ for $S_{n}$ and 467 as compared to $461 \mathrm{keV}$ for $S_{p}$. Inspecting the residuals in more detail we observe the well-known ability of the $\mathrm{L}_{1}$ norm estimation (i.e. RE) to confine in fewer observations the largest residuals. Therefore the residual 99th percentile is considerably larger for the RE adjustment (confinement of large residuals) whereas the residual 50th percentile is considerably smaller. In our case we can benefit much from this desirable property: if some of the measured energies contain gross errors they will be confined in those observations and not spread out through all observations (and results).

As we said before, it is also expected that the RE solution is more stable than the LS solution, i.e. some variations in the input data will have a larger impact on LS parameters than on RE parameters. For demonstration purposes we remove now the first $10 \%$ of the nuclei in the dataset (those with lower $A$ ) and compute again the LS and RE parameters. Results are shown in Table 3 for the one-neutron separation $S_{n}$ model (an analogous behavior is found for the one-proton separation $S_{p}$ model; for the sake of brevity we will restrict the rest of the explanation to the $S_{n}$ model).

Table 3. Parameters obtained for the $S_{n}$ model after LS adjustment for the complete dataset (column LS), the dataset after removal of the first $10 \%$ of data (column $\mathrm{LS}_{0.9}$ ) and variation in \% (column $\triangle \mathrm{LS}$ ). Analogously for RE results (respective columns $\mathrm{RE}, \mathrm{RE}_{0.9}$ and $\triangle \mathrm{RE}$ ).

\begin{tabular}{ccccccc}
\hline & $\begin{array}{c}\mathrm{LS} \\
(\mathrm{keV})\end{array}$ & $\begin{array}{c}\mathrm{LS}_{0.9} \\
(\mathrm{keV})\end{array}$ & $\begin{array}{c}\Delta \mathrm{LS} \\
(\%)\end{array}$ & $\begin{array}{c}\mathrm{RE} \\
(\mathrm{keV})\end{array}$ & $\begin{array}{c}\mathrm{RE}_{0.9} \\
(\mathrm{keV})\end{array}$ & $\begin{array}{c}\Delta \mathrm{RE} \\
(\%)\end{array}$ \\
\hline$a_{1}$ & 6294 & 4390 & 30 & 5227 & 4061 & 22 \\
$a_{2}$ & 3433 & 3664 & -7 & 3532 & 3685 & -4 \\
$a_{3}$ & 5850 & 5105 & 13 & 5364 & 4907 & 9 \\
$a_{\text {pair }}$ & 10587 & 11111 & -5 & 10874 & 11281 & -4 \\
$a_{\text {shell }}$ & 1512 & 1614 & -7 & 1552 & 1632 & -5 \\
\hline
\end{tabular}

We can clearly see that the impact of removing the dataset is larger for the LS estimated parameters (reaching the 30\%) than for the RE estimated parameters (reaching only 22\%). Being those variations quite large we must warn the reader, however, that their impact on the predictions made from them is not so dramatic, as we will later see.

Now let us use our model and estimated parameters to predict unmeasured energies and see if they respond, as expected, within accuracies of a few $\mathrm{keV}$. In the time interval between the 1995 Audi and Wapstra [12] dataset, which is the information that we have exclusively used in our analysis so far, and the AME2012 compilation [31] several one-neutron separation energies have been directly measured. They appear as experimentally determined values in the 2012 compilation [31] whereas in the 1995 dataset [12] appear as merely predicted values - for ${ }^{88} \mathrm{Tc},{ }^{89} \mathrm{Tc},{ }^{123} \mathrm{Ag},{ }^{140} \mathrm{I},{ }^{226} \mathrm{Rn},{ }^{227} \mathrm{Rn}$, ${ }^{228} \mathrm{Rn},{ }^{233} \mathrm{Ra}$, ${ }^{234} \mathrm{Ra}$ and ${ }^{235} \mathrm{Ac}$, e.g. - or simply not available (for ${ }^{222} \mathrm{Po}$ ).

We will now use the model for one-neutron separation energy - Eq. (1) - along with the estimated parameters by LS and RE, shown in Table 1, in order to obtain predictions for these currently known separation energies. We also compare the experimentally determined values with the predictions included in the 1995 Audi and Wapstra compilation [12] when available. Results are shown in Table 4 and Fig. 1. 
Table 4. Experimentally determined one-neutron energies $S_{n}$ and comparison with predictions by Audi and Wapstra (AW) [12], Eq. (1) with LS parameters and Eq. (1) with RE parameters. $\triangle \mathrm{AW}, \Delta \mathrm{LS}$ and $\triangle \mathrm{RE}$ show the respective differences between the energies obtained by each of these predictions and the experimental values.

\begin{tabular}{cccccccc}
\hline & $\begin{array}{c}\text { Experimental } \\
(\mathrm{keV})\end{array}$ & $\begin{array}{c}\mathrm{AW} \\
(\mathrm{keV})\end{array}$ & $\begin{array}{c}\mathrm{LS} \\
(\mathrm{keV})\end{array}$ & $\begin{array}{c}\mathrm{RE} \\
(\mathrm{keV})\end{array}$ & $\begin{array}{c}\Delta \mathrm{AW} \\
(\mathrm{keV})\end{array}$ & $\begin{array}{c}\Delta \mathrm{LS} \\
(\mathrm{keV})\end{array}$ & $\begin{array}{c}\Delta \mathrm{RE} \\
(\mathrm{keV})\end{array}$ \\
\hline${ }^{88} \mathrm{Tc}$ & 12060 & 11518 & 12114 & 11931 & -542 & 54 & -129 \\
${ }^{89} \mathrm{Tc}$ & 13780 & 12996 & 13970 & 13863 & -784 & 190 & 83 \\
${ }^{123} \mathrm{Ag}$ & 6510 & 6600 & 6530 & 6607 & 90 & 20 & 97 \\
${ }^{140} \mathrm{I}$ & 3210 & 3305 & 3411 & 3417 & 95 & 201 & 207 \\
${ }^{222} \mathrm{Po}$ & 5360 & & 5294 & 5357 & & -66 & -3 \\
${ }^{226} \mathrm{Rn}$ & 5858 & 5789 & 5516 & 5577 & -69 & -342 & -281 \\
${ }^{227} \mathrm{Rn}$ & 3933 & 3864 & 4009 & 4035 & -69 & 76 & 102 \\
${ }^{228} \mathrm{Rn}$ & 5714 & 5578 & 5315 & 5381 & -136 & -399 & -333 \\
${ }^{233} \mathrm{Ra}$ & 4246 & 4064 & 4047 & 4077 & -182 & -199 & -169 \\
${ }^{234} \mathrm{Ra}$ & 5500 & 5694 & 5337 & 5407 & 194 & -163 & -93 \\
${ }^{235} \mathrm{Ac}$ & 5555 & 5573 & 5541 & 5610 & 18 & -14 & 55 \\
${ }^{23 m s} \mathrm{dev}$. & & & & & 320 & 198 & 169 \\
\hline
\end{tabular}

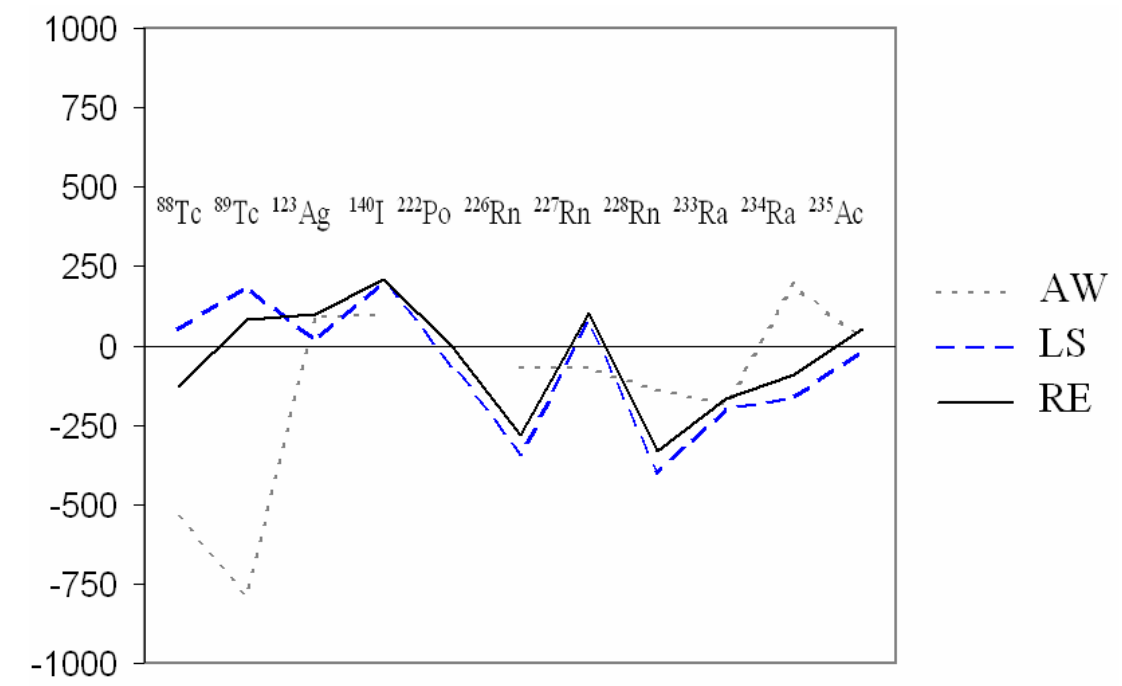

Figure 1. Differences of predictions by Audi and Wapstra, Eq. (1) with LS parameters and Eq. (1) with RE parameters with respect to the experimentally determined one-neutron energies $S_{n}$.

As it can be seen we obtain a slight improvement if we solve our model - Eq. (1) - by RE instead of LS. The root mean square deviation results in $169 \mathrm{keV}$ for the model solved by RE and $198 \mathrm{keV}$ for the model solved by LS, which show the excellent agreement of the predictions made by means of the simple model (1). Quite surprisingly, Audi and Wapstra predictions are considerably worse 
than those obtained by the simple model for two nuclei, ${ }^{88} \mathrm{Tc}$ and ${ }^{89} \mathrm{Tc}$, and yield a root mean square deviation of $320 \mathrm{keV}$.

Finally we will show that, as it was said before, the expected stability of the predictions made by the model solved by RE is higher than for the model solved by LS. If we use the parameters obtained for the dataset in which the first $10 \%$ of data has been removed, i.e. those shown under $\mathrm{LS}_{0.9}$ and $\mathrm{RE}_{0.9}$ in Table 3, the predicted values vary considerably less for the RE adjustment than for the LS adjustment. These results and variations are shown in Table 5.

Table 5. One-neutron predicted energies by Eq. (1) with LS parameters, Eq. (1) with LS parameters with the $10 \%$ data removed, Eq. (1) with RE parameters, Eq. (1) with RE parameters with the $10 \%$ data removed and respective variations.

\begin{tabular}{ccccccc}
\hline & $\begin{array}{c}\mathrm{LS} \\
(\mathrm{keV})\end{array}$ & $\begin{array}{c}\mathrm{LS}_{0.90} \\
(\mathrm{keV})\end{array}$ & $\begin{array}{c}\Delta \mathrm{LS} \\
(\mathrm{keV})\end{array}$ & $\begin{array}{c}\mathrm{RE} \\
(\mathrm{keV})\end{array}$ & $\begin{array}{c}\mathrm{RE}_{0.90} \\
(\mathrm{keV})\end{array}$ & $\begin{array}{c}\Delta \mathrm{RE} \\
(\mathrm{keV})\end{array}$ \\
\hline${ }^{88} \mathrm{Tc}$ & 12114 & 11866 & -248 & 11931 & 11802 & -129 \\
${ }^{89} \mathrm{Tc}$ & 13970 & 13856 & -114 & 13863 & 13833 & -30 \\
${ }^{123} \mathrm{Ag}$ & 6530 & 6654 & 124 & 6607 & 6692 & 84 \\
${ }^{140} \mathrm{I}$ & 3411 & 3380 & -31 & 3417 & 3375 & -42 \\
${ }^{222} \mathrm{Po}$ & 5294 & 5363 & 69 & 5357 & 5377 & 20 \\
${ }^{226} \mathrm{Rn}$ & 5516 & 5587 & 71 & 5577 & 5600 & 22 \\
${ }^{227} \mathrm{Rn}$ & 4009 & 4014 & 5 & 4035 & 4005 & -30 \\
${ }^{228} \mathrm{Rn}$ & 5315 & 5392 & 77 & 5381 & 5407 & 26 \\
${ }^{233} \mathrm{Ra}$ & 4047 & 4061 & 14 & 4077 & 4054 & -24 \\
${ }^{234} \mathrm{Ra}$ & 5337 & 5422 & 86 & 5407 & 5438 & 31 \\
${ }^{235} \mathrm{Ac}$ & 5541 & 5625 & 84 & 5610 & 5640 & 30 \\
$r m s \mathrm{dev}$. & & & 105 & & & 53 \\
\hline
\end{tabular}

Variations in the predicted values are considerably smaller for the model with parameters obtained by RE (being the root mean square deviation $53 \mathrm{keV}$ ) than for the model with parameters estimated by LS (whose root mean square deviation is $105 \mathrm{keV}$ ).

\section{Conclusion}

We have shown that both the predictions and the estimated parameters for the simple nucleon separation model (1) are considerably more stable, i.e. less dependent on the data used, for the RE adjustment than for LS estimation. Comparison against recently determined experimental data led us to conclude that predictions made by the simple model (1) solved by means of RE are a little better than those after LS estimation. Finally, we want to emphasize the usefulness of the results obtained by so a simple model, whose accuracy of some tens of $\mathrm{keV}$ is comparable to the accuracy that yield the best and much more complex models.

\section{References}


[1] Lunney D, Pearson J M and Thibault C 2003 Rev. Mod. Phys. 751021

[2] Weizsäcker CF 1935 Z. Phys. 96431

[3] Wang N, Liu M and Wu X Z 2010 Phys. Rev. C 81044322

[4] Garvey G T and Kelson I 1966 Phys. Rev. Lett. 16197

[5] Barea J, Frank A, Hirsch J G and Van Isacker P 2005 Phys. Rev. Lett. 94102501

[6] Tian J L, Wang N, Li C and Li JJ 2013 Phys. Rev. C 87014313

[7] Duflo J and Zuker A P 1995 Phys. Rev. C 52 R23

[8] Fu G J, Jiang H, Zhao Y M, Pittel S and Arima A 2010 Phys Rev C 82034304

[9] Jiang H, Fu G J, Zhao Y M and Arima A 2010 Phys Rev C 82054317

[10] Vogt K, Hartmann T and Zilges A 2001 Phys. Lett. B 517255

[11] Bao M, He Z, Zhao Y M and Arima A 2013 Phys. Rev. C 87044313

[12] Audi G and Wapstra H 1995 Nucl. Phys. A 595409

[13] Maronna R A, Martin D and Yohai V J 2006 Robust Statistics: Theory and Methods (Chichester: Wiley)

[14] Boscovich R J and Maire C 1755 De litteraria expeditione per pontificiam ditionem ad dimetiendos duos meridiani gradus et corrigendam mappam geographicam (Rome: Typographio Palladis)

[15] Laplace P S 1774 Savants Étranges 6621

[16] Box G E P 1953 Biometrika 40318

[17] Andrews D F, Bickel P J, Hampel F R, Huber P J, Rogers W H and Tukey J W 1972 Robust estimates of location: Surveys and advances (Princeton: Princeton University Press)

[18] Huber P J 1981 Robust statistics (New York: Wiley)

[19] Rousseeuw P J 1984 J. Am. Stat. Assoc. 79871

[20] Branham R L Jr 1982 Astron. J. 87928

[21] Wang L, Gordon M D and Zhu J 2006 Proc. Sixth Int. Conf. Data Mining 690

[22] Baselga S 2007 J. Surv. Eng. 133123

[23] Baselga S 2008 Comput. Math. Appl. 56709

[24] Baselga S and García-Asenjo L 2008 J. Navigation 61385

[25] Lin M H, Tsai J F and Yu C S 2012 Math. Probl. Eng. 756023

[26] Metropolis N, Rosenbluth M, Rosenbluth A, Teller A, Teller E 1953 J Chem Phys 211087

[27] Kirkpatrick S, Gelatt CD Jr, Vecchi MP 1983 Science 220671

[28] van Laarhoven P J M and Aarts E H L 1987 Simulated Annealing: Theory and Applications (Dordrecht: Reidel)

[29] Pardalos P M and Romeijn H E 2002 Handbook of Global Optimization (Dordrecht: Kluwer)

[30] Audi G, Wapstra A H and Thibault C 2003 Nucl. Phys. A 729337

[31] Wang M, Audi G, Wapstra A H, Kondev F G, MacCormick M, Xu X and Pfeiffer B 2012 Chin. Phys. C 361603 